\title{
Attenuated psychosis in primary caregiver of psychiatric patients: a cross-sectional study
}

\author{
Mahboobul Hasan Ansari ${ }^{1 *}$, Rakesh Kumar Gaur ${ }^{2}$, Suhail Ahmed Azmi ${ }^{3}$, \\ Ved Prakash Gupta ${ }^{4}$
}

\section{ABSTRACT}

Attenuated or transient Psychosis is experienced by a substantial proportion of the general population. Research also predicted that subjects perceiving psychotic-like experience are at high risk to develop psychotic spectrum disorder in the future. Aim The present study planned to estimate the psychotic-like experience or Attenuated psychosis in caregivers of psychotic patients attending the psychiatry department at JN Medical College, Aligarh Muslim University (AMU) Aligarh. In this study, we will also assess the relationship between disturbed sleep, perceived stress, and transient psychosis. Materials and methods: Purposive sampling was used to recruit all the subjects with informed written consent. All data were collected by applying a self-administered semi-structured proforma. Peters Delusions Inventory (PDI), Launay Slade Hallucination Scale-Revised (LSHS-R), Perceived Stress Questionnaire (PSQ), and Pittsburgh Sleep Quality Index (PSQI) General Health Questionnaire-12 (GHQ-12) on the subjects. Statistical analyses was done by using SPSS software 20 version. Result: Result shows the majority of caregivers belong to the age group 18-30 years (56.45\%). Maximum patients have the persecutory type of psychotic-like experience and among hallucinatory experience maximum subjects responded that "no matter how hard I try to concentrate; unrelated thoughts always creep into my mind". Delusion experiences were strongly positively correlated with hallucinatory experiences, perceived stress. Hallucinatory experiences were positively correlated with perceived stress poor sleep quality. Conclusion: It can be concluded that stress increases subclinical delusional and hallucinatory experiences. Though disturbed sleep is positively correlated with hallucination but not a delusion.

Keywords: Psychotic like experience, attenuated psychosis, primary caregiver, Perceived stress

\footnotetext{
${ }^{1}$ Department of Psychiatry, JN Medical College, AMU Aligarh, India

${ }^{2}$ Department of Psychiatry, JN Medical College, AMU Aligarh, India

${ }^{3}$ Department of Psychiatry, JN Medical College, AMU Aligarh, India

${ }^{4}$ Department of Psychiatry, JN Medical College, AMU Aligarh, India

*Responding Author
}

Received: June 19, 2020; Revision Received: June 23, 2020; Accepted: September 25, 2020

(C) 2020, M H Ansari, R K Gaur, S A Azmi \& V P Gupta; licensee IJIP. This is an Open Access Research distributed under the terms of the Creative Commons Attribution License (www.creativecommons.org/licenses/by/2.0), which permits unrestricted use, distribution, and reproduction in any Medium, provided the original work is properly cited. 


\section{Attenuated psychosis in primary caregiver of psychiatric patients: A cross-sectional study}

A

ttenuated psychosis (AP) is synonymous with psychotic-like experiences (PLE) and subclinical psychotic symptoms which can be described as a milder form of fullblown psychotic symptoms expressed in psychotic patients ${ }^{1}$. Mostly individual not reached the clinical threshold to be labeled as psychotic which require treatment. ${ }^{2}$ According to the continuum model of psychosis, symptoms of psychosis commonly found in individuals having no psychiatric diagnosis. ${ }^{1}$ However, persons with diagnosable clinical disorders exist at a certain point along this continuum. The majority of individuals in this continuum are made up of non-clinical cases while clinical cases of psychosis representing only a very small proportion of the total psychosis phenotype.

Recent research supported the idea that psychotic symptoms are experienced not only by patients suffering from psychiatric disorders but also by a large proportion of the apparently healthy population. The median prevalence AP is found to be $5-8 \%$ in the general population $^{3}$. Studies are suggested that AP is higher among adolescents aged group ${ }^{4}$. Attenuated psychosis (AP) may be an expression of schizotypy, which has been conceptualized as a non-clinical manifestation of the same underlying biological factors operating in schizophrenia and psychotic spectrum disorders.

Psychotic like experiences in the general population share an extensive range of risk factors with schizophrenia and therefore provide a useful phenotype in which to study the etiology of clinical psychosis. Some types of psychotic experiences, such as paranoid ideas, bizarre thinking, and perceptual abnormalities, may indicate a greater level of risk for psychotic disorder than other psychotic experiences, such as magical thinking.

Children and adolescents who experienced psychotic-like symptoms are at high risk to develop schizophrenia spectrum disorder in their adulthood ${ }^{5,6}$. A study conducted on the adult population revealed that $8 \%$ population converted into clinically psychotic 2 years later $^{7}$. This suggested that the population experiencing psychotic-like symptoms are of special concern.

Like schizophrenia, AP has several risk factors namely, victimization, substance use ${ }^{8}$, social situations like unemployment, the lower socio-economic background being unwed or divorced, and a higher rate of urbanicity ${ }^{9}$. A wide range of risk factors for schizophrenia has now been investigated in individuals who report PLEs and some striking similarities have emerged between the nonclinical and clinical populations, demonstrating construct validity between the clinical and non-clinical phenotypes.

Stressful life events are sufficient to cause pathology and illness behavior. Epidemiological studies conducted in the adult population revealed that, the mean prevalence of subclinical psychotic experiences at about $5 \%{ }^{10}$. For example, Scott et al. ${ }^{11}$ analyzing a sample of 1261 Australian adolescents, found that $8.4 \%$ of them reported having experienced some visual or auditory hallucinatory experience at some time. On his part, Horwood et $\mathrm{al}^{12}$. using a sample of 6455 English adolescents, found that 38.9\% scored on more than one item regarding psychotic experiences. In Spain, the interest in subclinical psychotic symptoms studies in the adolescent population has also increased. For example, Obiols et al ${ }^{13}$. using the Community Assessment of Psychic Experiences (CAPE) 20 in a sample of 777 adolescents, found that $44.1 \%$ reported at least one quasi-psychotic symptom and $19.7 \%$ manifested 3 or more experiences of this type. 
Caregivers of psychotic patients are at significantly higher psychological distress and share a hereditary character compared to a general medical condition. Since very scanty data are available on AP in Indian caregivers so this study is planned to conduct in the department of psychiatry, JN Medical College AMU Aligarh.

\section{Aims}

- The present study aims to estimate AP in caregivers of psychotic patients attending our medical college.

\section{Objectives}

1. To assess the distribution of AP in caregivers.

2. To determine the risk factors and socio-demographic factors associated with AP.

\section{MATERIALS AND METHODS}

This study was conducted in The Departments of Psychiatry, JN Medical College AMU Aligarh. The design of the study was cross-sectional in nature. All the caregivers accompanied by patients of schizophrenia spectrum disorder were recruited for the study and taken informed, written consent to participate in the study.

\section{Inclusion criteria}

1. Age $>18$ yrs of age

2. GHQ-12 score less than 3

3. Caregivers of patients who fulfilled the diagnostic criteria of schizophrenia spectrum disorder

4. Caregivers who gave informed consent

\section{Exclusion criteria}

1. Age $<18$ years or $>60$ years

2. Subjects suffering from seizure disorders.

3. Subjects suffering from any active medical disorder.

4. Subjects with a history of active psychiatric disorder, or those taking treatment for it.

\section{Procedure}

A sum of 310 subjects was assessed using pre-structured socio-demographic parameters, Peters Delusions Inventory (PDI), Launay-Slade Hallucination Scale-Revised (LSHS-R), General Health Questionnaire-12 (GHQ-12), Perceived Stress Questionnaire (PSQ) and Pittsburgh Sleep Quality Index (PSQI).

Observation

Socio-demographic variables:

\begin{tabular}{|l|l|l|}
\hline AGE GROUP & FREQUENCY & PERCENTAGE \\
\hline $18-30$ & 175 & $56.45 \%$ \\
\hline $31-40$ & 78 & $25.16 \%$ \\
\hline$>41$ & 57 & $18.38 \%$ \\
\hline Total & 310 & $100 \%$ \\
\hline
\end{tabular}

Majority of caregivers attending to our OPD were in the age group between 18-30 years and their mean age was found to be $36.46 \pm 8.26$. 
Attenuated psychosis in primary caregiver of psychiatric patients: A cross-sectional study

PDI-21 scoring

\begin{tabular}{|l|l|l|}
\hline QUESTIONNAIRES & \% ANSWERED "YES" & MEAN SEVERITY SCORE \\
\hline 1 & $59.4 \%$ & 6.6 \\
\hline 2 & $51.9 \%$ & 6.4 \\
\hline 3 & $37.7 \%$ & 8.2 \\
\hline 4 & $78.4 \%$ & 8.2 \\
\hline 5 & $49.8 \%$ & 7.4 \\
\hline 6 & $61.3 \%$ & 7.7 \\
\hline 7 & $59.4 \%$ & 7.3 \\
\hline 8 & $55.2 \%$ & 7.6 \\
\hline 9 & $48.1 \%$ & 7.7 \\
\hline 10 & $63.2 \%$ & 7.5 \\
\hline 11 & $54.5 \%$ & 7.2 \\
\hline 12 & $38.7 \%$ & 7.2 \\
\hline 13 & $49.4 \%$ & 7.4 \\
\hline 14 & $37.4 \%$ & 7.7 \\
\hline 15 & $48.1 \%$ & 7.3 \\
\hline 16 & $52 \%$ & 7.2 \\
\hline 17 & $39 \%$ & 6.9 \\
\hline 18 & $42.9 \%$ & 7.4 \\
\hline 19 & $59 \%$ & 7.2 \\
\hline 20 & $52.9 \%$ & 8.1 \\
\hline 21 & $34.8 \%$ & 7.1 \\
\hline
\end{tabular}

\section{LSHS-R Scoring}

\begin{tabular}{|l|l|}
\hline QUESTIONNAIRES & \% ANSWERED “YES" \\
\hline 1 & $72.2 \%$ \\
\hline 2 & $68.4 \%$ \\
\hline 3 & $71.6 \%$ \\
\hline 4 & $65.5 \%$ \\
\hline 5 & $55.2 \%$ \\
\hline 6 & $52.5 \%$ \\
\hline 7 & $58.4 \%$ \\
\hline 8 & $50.3 \%$ \\
\hline 9 & $47.7 \%$ \\
\hline 10 & $46.1 \%$ \\
\hline 11 & $48.4 \%$ \\
\hline & $41.3 \%$ \\
\hline
\end{tabular}

The highest percentage of patient-reported yes on "No matter how hard I try to concentrate, unrelated thoughts always creep into my mind" while $41.3 \%$ of subjects said that I have been troubled by hearing voices in my head.

\section{Correlations:}

\begin{tabular}{|l|l|l|l|}
\hline \multicolumn{2}{|l|}{ LSHS-R } & PSQ & PSQI \\
\hline PDI-21 & $0.384 * *$ & $0.306 * *$ & 0.026 \\
\hline \multicolumn{4}{|l|}{} \\
\hline LSHS-R & PDI & PSQ & PSQI \\
\hline
\end{tabular}




\section{Attenuated psychosis in primary caregiver of psychiatric patients: A cross-sectional study}

Delusional experiences are positively related to Hallucinatory experiences perceived stress and are highly statistically significant. Hallucinatory experiences are positively correlated with perceived stress and sleep quality index and statistically significant.

\section{DISCUSSION}

In this study, we included only those subjects who came to us along with their patient as caregivers. Among them, a large proportion (56.45\%) belong to the age group 18-30 years. In this study, we observed that subclinical psychotic experiences are quite common in caregivers of psychiatric patients. Among delusional experiences around 78.4\% of patient's caregivers experienced that "they are being persecuted in some way" the highest experienced PDI item, only 34.8\% caregivers felt that they are a robot or zombie without their will" a least delusional experienced. As we know, caregivers of psychotic patients are much more disturbed compared to caregivers of medical illness. Much research conducted in the past provided strong evidence of the relation between anxiety and persecutory feeling ${ }^{14}$. Evidence of Anxiety and its relation to paranoia has been proved for a long time in the west $^{15}$.

Our finding is higher than the previous observations in which the incidence of hallucination was found to be $10-15 \%$ in the general population ${ }^{16}$. Since our finding is not under the influence of any psychoactive substance. So this study further supports the continuum model of psychosis, which suggested that initially brief and AP commonly present in a healthy population but in the least severe form. Later subclinical psychosis persists with increased severity. In the last, psychotic disorder develops with clinically distressful conditions with functional impairment. The researcher also confirmed that the difference between nonclinical and clinical psychotic symptoms is quantitative rather qualitative ${ }^{17}$.

This study is among the few conducted in India which established the relationship with the experience of psychosis and sleep problems. In our results, there was a strong positive correlation was found between poor sleep quality and delusional and hallucinatory experiences. A bidirectional link has been established between sleep problems and psychotic experiences. Our finding is further supported by both model of psychosis ${ }^{18}$, in which sleep disturbance and mental health interact reciprocally via changes in cognitive and daily activity ${ }^{19}$.

In the present study, we observed the positive correlation between perceived stress questionnaire and both the component of psychosis i.e delusion like experience and hallucination like experiences. This is further supported by the study conducted in the past which proved persecutory ideation has been associated with a high level of distress ${ }^{20}$.

\section{CONCLUSION}

Our finding suggested that caregivers of psychotic patients encounter much more psychoticlike experiences. So we need to explore the perceived stress and poor quality of sleep in the caregivers of the patient as early as possible to intervene time. If we will be able to find the magnitude of sub psychotic symptoms we can intervene time to stop the progression of the psychotic phenomenon by applying appropriate measures.

\section{Limitation}

Since this study was conducted only on the caregiver who were accompanying psychotic patients so it did not measure the degree of relation between subjects and patients. Due to this reason, genetic factors could not be excluded in our study. Not the inclusion of a control 
group is another demerit of our study. Childhood trauma and personality are also risk factors for PLE which was not considered in this study.

\section{REFERENCES}

1. Bentall, R.P., 2003. Madness Explained: Psychosis and Human Nature. Penguin Books Ltd., London.

2. Kwapil TR, Barrantes Vidal N, Silvia PJ. The dimensional structure of the Wisconsin schizotypy scales: Factor identifi cation and construct validity. Schizophr Bull 2008;34:444-57.

3. Van Os J, Linscott RJ, Myin-Germeys I, Delespaul P, Krabbendam L (2009). A systematic review and meta-analysis of the psychosis continuum: evidence for a psychosis proneness-persistence-impairment model of psychotic disorder. Psychological Medicine 39, 179-195.

4. Laurens KR, Hodgins S, Maughan B, Murray RM, Rutter ML, Taylor EA (2007). Community screening for psychotic-like experiences and other putative antecedents of schizophrenia in children aged 9-12 years. Schizophrenia Research 90, 130-146.

5. Poulton R, Caspi A, Moffitt TE, Cannon M, Murray R, Harrington H (2000). Children's self-reported psychotic symptoms and adult schizophreniform disorder : a 15-year longitudinal study. Archives of General Psychiatry 57, 1053-1058.

6. Welham J, Scott J, Williams G, Najman J, Bor W, O'Callaghan M, McGrath J (2009). Emotional and behavioural antecedents of young adults who screen positive for non-affective psychosis: a 21-year birth cohort study. Psychological Medicine 39, 625-634.

7. Hanssen M, Maarten B, Rob B, Vollebergh W, van Os J (2005). The incidence and outcome of subclinical psychotic experiences in the general population. British Journal of Clinical Psychology 44, 181-191.

8. Mackie CJ, Castellanos N, Conrod PJ (2010). Developmental trajectories of psychotic-like experiences across adolescence: impact of victimization and substance abuse. Psychological Medicine

9. Scott J, Chant D, Andrews G, McGrath J (2006). Psychotic-like experiences in the general community: the correlates of CIDI psychosis screen items in an Australian sample. Psychological Medicine 36, 231-238.

10. van Os J, Linscott RJ, Myin-Germeys I, Delespaul P, Krabbendam L. A systematic review and meta-analysis of the psychosis continuum: Evidence for a psychosis proneness-persistence-impairment model of psychotic disorder. Psychol Med 2009;39:179-95.

11. Scott J, Martin G, Bor W, Sawyer M, Clark J, McGrath J. The prevalence and correlates of hallucinations in Australian adolescents: Results from a national survey. Schizophr Res 2009;109:179-85.

12. Horwood J, Thomas K, Duffy L, Gunnell D, Hollis C, Lewis G,et al. Frequency of psychosis-like symptoms in a non-clinical population of 12 years olds: Results from the Alspac birth cohort. Schizophr Res 2008;98:77-8

13. Obiols JE, Barragán M, Vicens J, Navarro JB. Síntomas seudo-psicóticos en adolescentes de la población general. Rev Psicopatol Psicol Clín 2008;13:205 17.

14. Freeman, D. (2008). Studying and treating schizophrenia using virtual reality: a new paradigm. Schizophrenia bulletin, 34(4), 605-610.

15. Freeman, D., \& Fowler, D. (2009). Routes to psychotic symptoms: trauma, anxiety and psychosis-like experiences. Psychiatry research, 169(2), 107-112.

16. Sommer, I. E., Daalman, K., Rietkerk, T., Diederen, K. M., Bakker, S., Wijkstra, J., \& Boks, M. P. (2010). Healthy individuals with auditory verbal hallucinations; who 
are they? Psychiatric assessments of a selected sample of 103 subjects. Schizophrenia bulletin, 36(3), 633-641.

17. Castiajo, P., \& Pinheiro, A. P. (2017). On "Hearing" voices and "Seeing" things: probing hallucination predisposition in a Portuguese nonclinical sample with the launay-slade hallucination scale-revised. Frontiers in psychology, 8, 1138.

18. Freeman, D., Startup, H., Dunn, G., Černis, E., Wingham, G.et al. (2013). The interaction of affective with psychotic processes: a test of the effects of worrying on working memory, jumping to conclusions, and anomalies of experience in patients with persecutory delusions. Journal of psychiatric research, 47(12), 1837-1842.

19. Waite, F., Evans, N., Myers, E., Startup, H., Lister, R., Harvey, A. G., \& Freeman, D. (2016). The patient experience of sleep problems and their treatment in the context of current delusions and hallucinations. Psychology and Psychotherapy: Theory, Research and Practice, 89(2), 181-193.

20. Freeman D, Garety PA, Kuipers E. Persecutory delusions: developing the understanding of belief maintenance and emotional distress. Psychol Med. 2001;31(7):1293-1306.

\section{Acknowledgements}

The author appreciates all those who participated in the study and helped to facilitate the research process.

\section{Conflict of Interest}

The author declared no conflict of interest.

How to cite this article: M H Ansari, R K Gaur, S A Azmi \& V P Gupta (2020). Attenuated psychosis in primary caregiver of psychiatric patients: A cross-sectional study. International Journal of Indian Psychology, 8(3), 17-23. DIP:18.01.003/20200803, DOI:10.25215/0803.003 